\title{
Anti-bacterial antibodies in multiple myeloma patients at disease presentation, in response to therapy and in remission: implications for patient management
}

\author{
Ilaria J. Chicca (1)', Jennifer L. J. Heaney ${ }^{1}$, Gulnaz lqbal', Janet A. Dunn², Stella Bowcock $\mathbb{E}^{3}$, Guy Pratt ${ }^{4}$, Kwee L. Yong ${ }^{5}$, \\ Timothy D. Planche $\mathbb{D}^{6}$, Alex Richter ${ }^{1}$ and Mark T. Drayson (1)
}

\begin{abstract}
Multiple myeloma (MM) is associated with increased risk of infection, but little is known regarding antibody levels against specific bacteria. We assessed levels of polyclonal immunoglobulin and antibacterial antibodies in patients recruited to the TEAMM trial, a randomised trial of antibiotic prophylaxis at the start of anti-myeloma treatment. Polyclonal IgG, IgA and IgM levels were below the reference range in $71 \%, 83 \%$ and $90 \%$ of $838 \mathrm{MM}$ patients at diagnosis. Anti-vaccine targeted tetanus toxoid antibodies were protective in $95 \%$ of 193 healthy controls but only $41 \%$ of myeloma patients. In healthy controls, protective antibodies against 6/12 pneumococcal serotypes, haemophilus and meningococcus A were present in $67 \%, 41 \%$ and $56 \%$ compared to just $15 \%, 21 \%$ and $17 \%$ of myeloma patients. By 1 year, myeloma patients Ig G levels had recovered for $57 \%$ of patients whilst the proportion with protective levels of IgG against thymus-dependent protein antigen tetanus toxoid had changed little. In contrast the proportions of patients with protective levels against thymus independent polysaccharide antigens pneumococcus, haemophilus and meningococcus had fallen from 15 to $7 \%, 21$ to $0 \%$ and 17 to $11 \%$. Findings highlight the need for strategies to protect patients against bacterial infections during therapy and vaccination programmes during remission.
\end{abstract}

\section{Introduction}

Multiple myeloma (MM) arises from the malignant proliferation of a single clone of plasma cells in the bone marrow and accounts for over $10 \%$ of haematological malignancies ${ }^{1-3}$. With the average age at diagnosis being 70 years MM causes anaemia, skeletal fractures, renal failure and profound cellular and humoral immunodeficiency $^{1-3}$. Advances in anti-myeloma therapy have improved overall survival (OS) to $50 \%$ at 5 years but infection contributes to death in a fifth of patients with

\footnotetext{
Correspondence: Mark T. Drayson (M.T.Drayson@bham.ac.uk)

${ }^{1}$ Institute of Immunology and Immunotherapy, Clinical Immunology Service, University of Birmingham, Birmingham, UK

${ }^{2}$ Warwick Clinical Trial Unit, University of Warwick, Coventry, UK

Full list of author information is available at the end of the article
}

myeloma ${ }^{1,2,4-7}$. The risk of infection is greatest in the first 3 months after diagnosis, with a third of patients suffering serious bacterial infection, and infection contributing to half of early mortality ${ }^{8-10}$. The most common causes of infection are encapsulated extracellular bacteria such as Streptococcus pneumoniae and Haemophilus influenza ${ }^{8}$. Although novel agents and intensive therapeutic strategies have improved survival rates for myeloma, antimyeloma therapy may further suppress patient's immunological functions ${ }^{7}$.

Humoral immunodeficiency in MM is characterised by polyclonal IgM, IgG and IgA levels below the normal range in $85 \%$ of patients (immunoparesis) ${ }^{11-13}$. Lower levels of polyclonal IgG and IgA have been reported to be predictive of blood stream infections in the first few 
months from MM diagnosis and serious infection episodes during MM remission ${ }^{14,15}$. However, Danish registry analysis of $2557 \mathrm{MM}$ patients during 6 months from diagnosis showed tumour burden and renal impairment but not immunoparesis were risk factors for infection ${ }^{16}$.

The level of suppression of polyclonal immunoglobulin in patients is inversely correlated with the level of Mprotein and with MM stage, indicating increased suppression is identified in more aggressive forms of disease $^{12,13,17}$. In recent UK myeloma trials median progression-free survival (PFS) was 57\%, 39\% and 36\% longer for patients with normal IgM, IgG and IgA levels, respectively ${ }^{12}$. The depth of IgM suppression, but not the depth of IgG or IgA suppression, was prognostic for survival: the most severely suppressed IgM tertile of patients OS was 0.9 years shorter than those in the top tertile, and 2.6 years shorter than OS of those with normal IgM levels

Much is known about the significance of total levels of polyclonal immunoglobulins in MM with respect to survival but as we have found they are not strongly predictive of infection ${ }^{12}$. To our knowledge, the significance of antibody levels specific to different bacterial antigenic targets at diagnosis and following modern MM therapies has yet to be characterised. A clear picture of patients' immunological status may help stratify them for risk of specific infections during induction therapy and may be of prognostic value for OS and PFS. Also, understanding the timeframe of immunoglobulin recovery may have an impact on the clinical management of patients.

We assessed polyclonal immunoglobulin levels and those directed against pertinent bacterial antigens at disease presentation and after 1 year in a large patient population from the UK Tackling-Early-Morbidity-andMortality-in-Myeloma (TEAMM) trial that had been designed to investigate the effect of antibiotic prophylaxis on infections and deaths ${ }^{18}$.

\section{Methods}

\section{TEAMM Trial and patients}

The TEAMM trial was a multicentre randomised, double-blind, placebo-controlled trial in newly diagnosed myeloma patients randomised to receive Levofloxacin or placebo for 12 weeks at the start of anti-myeloma treatment. Follow-up was 4-weekly to 16 weeks and again at 1 year. The primary outcome was time to first febrile episode or death in the first 12 weeks from start of trial treatment. Secondary outcomes included number of infections, deaths, healthcare-associated organism carriage and invasive infections, and $\mathrm{OS}^{18}$.

The TEAMM trial recruited between August 2012 and April 2016, 977 patients aged $\geq 21$ years with newly diagnosed symptomatic myeloma, and within 14 days of starting active myeloma treatment. Treatment allocation was blinded and balanced by centre, estimated glomerular filtration rate (eGFR) and intention to give high-dose chemotherapy with autologous stem cell transplant. The 838 included in the present investigation out of 977 patients in the trial represent patients with sufficient serum sample available for analysis. Details of treatment, however, are limited to intended treatments at baseline as the trial did not record treatment delivered, with the exception of steroid usage. The trial (ISRCTN registration No. 51731976) had approval from the UK Coventry \& Warwickshire Multi-Research Ethics Committee and all patients provided written informed consent. Serum samples from 193 healthy adult donors for determining adult ranges for the assays were from NHS Blood and Transplant, Birmingham, UK (NHSBT; age range 17-65 years).

\section{Laboratory analysis}

Serum from diagnosis was analysed centrally at the Clinical Immunology Service (Birmingham, UK) by protein electrophoresis, densitometry and immunofixation for M-protein quantification and characterisation. Serum IgG, IgA and IgM, and kappa and lambda free light chains (sFLC) levels were quantified by turbidimetry. Patients were characterised as having one of the following multiple myelomas: IgG, IgA, IgM, IgD, light chain only (LCO), non-secretory (NS) or oligosecretory; with either kappa or lambda monoclonal light chain. Patients were classified as being below, within or above normal range (NR) for polyclonal immunoglobulins based upon 5th-95th centile ranges of adults aged over 45 years in the UK reported by Protein Reference Units: IgG 6-16 g/l; IgA 0.8-4 g/l; and IgM 0.5-2 g/l.

The serum from 838 MM patients (aged 35-90) enrolled in the TEAMM trial was analysed at disease presentation and after 1 year from first trial visit. The concentration of 19 anti-bacterial antibodies were quantified using a multiplexed Luminex assay, described in detail previously ${ }^{19}$. IgG antibody levels were measured against 12 pneumococcal (Pn) serotypes, 4 meningococcal (Men) serotypes, Haemophilus influenzae type b (Hib) polysaccharide, and tetanus and diphtheria toxoids. Protective thresholds for IgG were: $0.35 \mu \mathrm{g} / \mathrm{ml}$ for Pn serotypes $^{20}, 2 \mu \mathrm{g} / \mathrm{ml}$ for Men serotypes ${ }^{21}, 1 \mu \mathrm{g} / \mathrm{ml}$ for Hib polysaccharide $^{22}$, and $0.1 \mathrm{IU} / \mathrm{ml}$ for diphtheria and tetanus $^{23}$. Streptococcus pneumoniae capsular polysaccharides were obtained from the American Type Culture Collection (Manassas, Virginia, USA), Tetanus toxoid was obtained from Quadratech (Epsom, UK), Neisseria meningitidis and Hib capsular polysaccharides, and diphtheria toxoid were from the National Institute for Biological Standards and Control (Potters Bar, UK). Briefly, polysaccharide antigens were pre-conjugated to poly-L-lysine (Sigma-Aldrich, Darmstadt, Germany) and purified. Poly-L-lysine-conjugated antigens and toxoids 
were conjugated to carboxylated microspheres (Bio-plex Systems, BioRad Laboratories, Hercules, California, USA), each corresponding to a different bead region, through a two-steps carbodiimide reaction. Beads were resuspended in phosphate-buffered saline (PBS) 0.1\% Bovine Serum Albumin (BSA), $0.05 \%$ sodium azide, counted with a haemocytometer and stored in the dark at $4{ }^{\circ} \mathrm{C}$. The analysis of serum samples for 19 antigens (19-plex) on multi-beads was performed in a 96-well filter plate. A 12 point standard curve was obtained following a 12-fold 1:2 dilution of standard serum 007 in PBS 1\% BSA $0.05 \%$ Tween 20 containing $5 \mu \mathrm{g} / \mathrm{ml}$ pneumococcal cell wall polysaccharide (Statens Serum Institute, Copenhagen, Denmark). Serum samples were diluted 1:100 using PBS $1 \%$ BSA $0.05 \%$ Tween containing $5 \mu \mathrm{g} / \mathrm{ml}$ pneumococcal cell wall polysaccharide and $5 \mu \mathrm{g} / \mathrm{ml}$ pneumococcal serotype $22 \mathrm{~F}$ to prevent unspecific binding. Human serum, pooled serum and serum deprived of IgGs were used as controls. In all, $200 \mu \mathrm{l}$ of PBS $0.05 \%$ Tween 20 were aspirated through the filter of each well to pre-wet the filter. Twenty-five microlitres of bead-conjugated antibody mix were added to each well and washed twice with PBS $0.05 \%$ Tween 20. Twenty-five microlitres of test samples were added to the plate and incubated at room temperature covered from light for $1 \mathrm{~h}$ on an orbital shaker at $500 \mathrm{rpm}$. After washing, $100 \mu \mathrm{l}$ of mouse antihuman IgG phycoerythrin-conjugated antibody (Southern Biotech, Birmingham, Alabama, USA) diluted 1:200 were added and left in incubation at room temperature covered from light for $30 \mathrm{~min}$ on an orbital shaker at $500 \mathrm{rpm}$. Wells were washed twice and beads were resuspended in $125 \mu \mathrm{l}$ PBS 0.05\% Tween 20 for the reading. Fluorescent intensity was measured with a Luminex-200 instrument (BioRad Laboratories) and data analysis was performed with Bio-plex Manager 6.1 software (BioRad Laboratories). Polyclonal IgG, IgA and IgM were quantified by turbidimetry. Antibody levels in patients with MM were compared to a cohort of 193 healthy adult blood transfusion service donors ${ }^{19}$.

\section{Statistical analysis}

Statistical analyses were performed using Prism v. 8 software (GraphPad, San Diego, California, USA) and SAS ${ }^{\circledR}$ (version 9.3; SAS Institute Inc., Cary, NC, USA) software. Statistical significance of the results was determined using the Chi-Square test to identify differences in categorical variables between groups, Wilcoxon's rank test to compared differences between paired data and the Mann-Whitney test or Kruskal-Wallis test to analyse differences between unpaired data. Correlations between biomarkers and outcomes measures were investigated using Spearman's rank test. Infection rates and febrile episodes were investigated based on severity of polyclonal immunoglobulin suppression. Patients were split into groups based on normal, slightly reduced and severely reduced polyclonal immunoglobulins. Incidence of infection (yes/no) or febrile episode (yes/no) in the first 12 weeks was compared between groups polyclonal immunoglobulin groups using Fisher's exact test.

Analysis of time to first infection within 12 weeks was carried out using a log-rank comparison, starting from the date the patient started trial treatment to the date of infection, or to a censor date for those with no infections. Cox regression analyses were performed to determine independent predictors of febrile episode or death. Patient and disease characteristics were considered, including polyclonal IgM, polysaccharide antigens, tetanus and diphtheria toxoids. Overall survival was calculated from date of starting trial treatment to the date of death or censor date for those alive up to 12 months.

In addition to TEAMM treatment allocation (Levofloxacin or placebo), prophylactic Septrin was evaluated as an independent predictor of febrile episode or death. Patients were ineligible for the TEAMM trial if receiving other prophylactic antibiotic treatment, excluding pneumocystis prophylaxis if regarded as essential. Prophylactic co-trimoxazole $960 \mathrm{mg}$ (Septrin) three times per week to prevent pneumocysistis was allowed and given to 314 patients within the TEAMM trial due to its wide use in the UK but was not part of stratification or randomisation. Results of the TEAMM trial showed a benefit in reducing febrile episodes for patients taking septrin prophylaxis, a benefit for levofloxacin prophylaxis and the greatest benefit for patients who took both antibiotics as prophylaxis compared to the population receiving no antibiotic prophylaxis.

\section{Results}

\section{Patient characteristics}

At entry to the trial patients were stratified according to intention to treat intensively with an autologous stem cell rescue procedure (transplant eligible TE) or for older frailer patients to receive non-intensive therapy (Transplant non-eligible $\mathrm{TNE})^{24}$. Patient characteristics are presented in Table 1 split by intention to give intensive treatment (456 TE patients) or not (380 TNE patients). TE patients were younger, had better performance status, renal function and earlier stage myeloma disease. There was no difference in neutrophil counts with $9 \%$ having levels $<1.8 \times 10^{9} / 1$ but lymphopaenia $\left(<1.2 \times 10^{9} / \mathrm{l}\right)$ was commoner in TNE patients. $99 \%$ of all patients treatment included an immunomodulatory drug or proteasome inhibitor as anti-myeloma therapy and bisphosphonates.

\section{Polyclonal immunoglobulin levels are low at MM diagnosis and recover significantly by 1 year}

Serum polyclonal IgG, IgA and IgM were measured in TEAMM patients without an IgG, IgA and IgM 
Table 1 Patient characteristics by intention to give intensive treatment.

\begin{tabular}{|c|c|c|c|c|c|c|c|}
\hline \multirow[b]{3}{*}{ Factor } & \multirow[b]{3}{*}{ Grouping } & \multicolumn{4}{|c|}{ Intention to give intensive treatment } & & \\
\hline & & \multicolumn{2}{|l|}{$\begin{array}{l}\text { No } \\
n=\mathbf{3 8 0}\end{array}$} & \multicolumn{2}{|l|}{$\begin{array}{l}\text { Yes } \\
n=456\end{array}$} & \multicolumn{2}{|c|}{$\begin{array}{l}\text { Total } \\
n=836\end{array}$} \\
\hline & & $N$ & $\%$ & $N$ & $\%$ & $N$ & $\%$ \\
\hline Age (years) & Median (IQR) & $75(70-79)$ & $61(54-66)$ & $67(60-75)$ & & & \\
\hline \multirow[t]{2}{*}{ Gender } & Male & 236 & 62 & 293 & 64 & 529 & 63 \\
\hline & Female & 144 & 38 & 163 & 36 & 307 & 37 \\
\hline \multirow[t]{6}{*}{ Ethnicity } & White & 356 & 94 & 412 & 90 & 768 & 92 \\
\hline & Mixed & 1 & $<1$ & 2 & $<1$ & 3 & $<1$ \\
\hline & Asian or British Asian & 3 & 1 & 17 & 4 & 20 & 2 \\
\hline & Black or Black British & 20 & 5 & 23 & 5 & 43 & 5 \\
\hline & Chinese or Other & 0 & 0 & 1 & $<1$ & 3 & $<1$ \\
\hline & Missing & 0 & 0 & 1 & $<1$ & 1 & $<1$ \\
\hline Performance & 0 & 91 & 24 & 201 & 44 & 292 & 35 \\
\hline status at & 1 & 170 & 45 & 169 & 37 & 339 & 41 \\
\hline \multirow[t]{4}{*}{ Randomisation } & 2 & 80 & 21 & 54 & 12 & 134 & 16 \\
\hline & 3 & 27 & 7 & 24 & 5 & 51 & 6 \\
\hline & 4 & 1 & $<1$ & 3 & 1 & 4 & $<1$ \\
\hline & Missing & 11 & 3 & 5 & 1 & 16 & 2 \\
\hline International Staging & Stage I & 64 & 17 & 121 & 26 & 185 & 22 \\
\hline \multirow[t]{3}{*}{ System } & Stage II & 117 & 31 & 182 & 40 & 299 & 36 \\
\hline & Stage III & 125 & 33 & 99 & 22 & 224 & 27 \\
\hline & Missing & 74 & 19 & 54 & 12 & 128 & 15 \\
\hline \multirow[t]{3}{*}{ eGFR } & $>50 \mathrm{ml} / \mathrm{min}$ & 246 & 65 & 381 & 84 & 627 & 75 \\
\hline & $20-50 \mathrm{ml} / \mathrm{min}$ & 101 & 26 & 64 & 14 & 165 & 20 \\
\hline & $<20 \mathrm{ml} / \mathrm{min}$ & 33 & 9 & 11 & 2 & 44 & 5 \\
\hline \multirow[t]{3}{*}{ Prior infection } & Clostridium-Difficile & 2 & 1 & 1 & $<1$ & 3 & $<1$ \\
\hline & MRSA & 6 & 2 & 5 & 1 & 11 & 1 \\
\hline & ESBL coliforms & 2 & 1 & 6 & 1 & 8 & 1 \\
\hline Anti-infectives & No & 237 & 63 & 323 & 71 & 560 & 67 \\
\hline \multirow[t]{2}{*}{ in month prior } & Yes & 62 & 16 & 72 & 16 & 134 & 16 \\
\hline & Missing & 81 & 21 & 61 & 13 & 142 & 17 \\
\hline Steroids 14 days prior to randomisation & Yes & 180 & 47 & 243 & 53 & 423 & 51 \\
\hline \multirow[t]{3}{*}{ Corticosteroids } & Prednisolone & 23 & 6 & 11 & 2 & 34 & 4 \\
\hline & Dexamethasone & 158 & 42 & 234 & 51 & 392 & 47 \\
\hline & Other & 1 & $<1$ & 1 & $<1$ & 2 & $<1$ \\
\hline Planned anti-myeloma & Thalidomide based & 185 & 49 & 178 & 39 & 363 & 43 \\
\hline \multirow[t]{2}{*}{ treatment } & Bortezomib based & 120 & 32 & 146 & 32 & 266 & 32 \\
\hline & Lenalidomide based & 64 & 17 & 57 & 13 & 121 & 14 \\
\hline
\end{tabular}


Table 1 continued

\begin{tabular}{|c|c|c|c|c|c|c|c|}
\hline \multirow[b]{3}{*}{ Factor } & \multirow[b]{3}{*}{ Grouping } & \multicolumn{4}{|c|}{ Intention to give intensive treatment } & & \\
\hline & & \multicolumn{2}{|c|}{$\begin{array}{l}\text { No } \\
n=380\end{array}$} & \multicolumn{2}{|c|}{$\begin{array}{l}\text { Yes } \\
n=456\end{array}$} & \multicolumn{2}{|c|}{$\begin{array}{l}\text { Total } \\
n=836\end{array}$} \\
\hline & & $N$ & $\%$ & $N$ & $\%$ & $N$ & $\%$ \\
\hline & Lenalidomide \& Carfilzomib based & 3 & 1 & 71 & 16 & 74 & 9 \\
\hline & Other & 8 & 2 & 4 & 1 & 12 & 1 \\
\hline Bisphosphonate & Not given & 65 & 17 & 39 & 9 & 104 & 12 \\
\hline Status at & Given/will be given & 313 & 82 & 414 & 91 & 727 & 87 \\
\hline Randomisation & Missing & 2 & 1 & 3 & 1 & 5 & 1 \\
\hline \multirow[t]{5}{*}{ Bisphosphonate } & Zolendronate & 194 & 51 & 293 & 64 & 487 & 58 \\
\hline & Pamidronate & 94 & 25 & 96 & 21 & 190 & 23 \\
\hline & Clodronate & 19 & 5 & 14 & 3 & 33 & 4 \\
\hline & Other & 3 & 1 & 5 & 1 & 8 & 1 \\
\hline & Missing & 70 & 18 & 48 & 11 & 118 & 14 \\
\hline Prophylactic anti- & No & 209 & 55 & 205 & 45 & 414 & 50 \\
\hline viral/fungal & Yes & 171 & 45 & 251 & 55 & 422 & 50 \\
\hline Corrected calcium & $<2.5$ & 273 & 72 & 317 & 70 & 590 & 70 \\
\hline \multirow[t]{3}{*}{$(\mu \mathrm{mol} / \mathrm{l})$} & $2.5-2.75$ & 78 & 20 & 97 & 21 & 175 & 21 \\
\hline & $>2.75$ & 15 & 4 & 33 & 7 & 48 & 6 \\
\hline & Missing & 14 & 4 & 9 & 2 & 23 & 3 \\
\hline Evidence of bone disease & Yes & 265 & 70 & 336 & 74 & 601 & 72 \\
\hline \multirow[t]{5}{*}{ Site of bone disease } & Vertebral fracture/collapse & 115 & 30 & 114 & 25 & 229 & 27 \\
\hline & Lytic lesions & 180 & 47 & 238 & 52 & 418 & 50 \\
\hline & Fractured rib & 22 & 6 & 27 & 6 & 49 & 6 \\
\hline & Osteoporosis & 38 & 10 & 23 & 5 & 61 & 7 \\
\hline & Other fracture & 28 & 7 & 44 & 10 & 72 & 9 \\
\hline \multirow[t]{4}{*}{$\mathrm{Sb} 2 \mathrm{~m}(\mathrm{mg} / \mathrm{l})$} & $\leq 4$ & 111 & 29 & 216 & 47 & 327 & 39 \\
\hline & $4-8$ & 119 & 31 & 139 & 30 & 258 & 31 \\
\hline & $>8$ & 76 & 20 & 48 & 11 & 124 & 15 \\
\hline & Missing & 74 & 20 & 53 & 12 & 127 & 15 \\
\hline \multirow[t]{4}{*}{ Anaemia ( $\mathrm{Hb} \mathrm{g} / \mathrm{dl})$} & $<7.5$ & 11 & 3 & 9 & 2 & 20 & 2 \\
\hline & $7.5-10$ & 144 & 38 & 137 & 30 & 281 & 34 \\
\hline & $>10$ & 221 & 58 & 310 & 68 & 531 & 64 \\
\hline & Missing & 4 & 1 & 0 & 0 & 4 & $<1$ \\
\hline Thrombocytopenia & $\leq 150$ & 63 & 17 & 62 & 14 & 125 & 15 \\
\hline \multirow[t]{2}{*}{ (platelets $\times 10^{9} / \mathrm{l}$ ) } & $>150$ & 313 & 82 & 393 & 86 & 706 & 84 \\
\hline & Missing & 4 & 1 & 1 & $<1$ & 5 & 1 \\
\hline \multirow[t]{2}{*}{ Neutrophils $\left(\times 10^{9} / \mid\right)$} & $<1.8$ & 36 & 9 & 39 & 9 & 75 & 9 \\
\hline & 1.8 to 3 & 106 & 28 & 132 & 29 & 238 & 28 \\
\hline
\end{tabular}




\begin{tabular}{|c|c|c|c|c|c|c|c|}
\hline \multirow[b]{3}{*}{ Factor } & \multirow[b]{3}{*}{ Grouping } & \multicolumn{4}{|c|}{ Intention to give intensive treatment } & & \\
\hline & & \multicolumn{2}{|c|}{$\begin{array}{l}\text { No } \\
n=380\end{array}$} & \multicolumn{2}{|c|}{$\begin{array}{l}\text { Yes } \\
n=456\end{array}$} & \multicolumn{2}{|c|}{$\begin{array}{l}\text { Total } \\
n=836\end{array}$} \\
\hline & & $N$ & $\%$ & $N$ & $\%$ & $N$ & $\%$ \\
\hline & $>3$ & 232 & 61 & 284 & 62 & 516 & 62 \\
\hline & Missing & 6 & 2 & 1 & $<1$ & 7 & 1 \\
\hline \multirow[t]{4}{*}{ Lymphocytes } & $<1.2$ & 104 & 27 & 104 & 23 & 208 & 25 \\
\hline & $1.2-1.8$ & 252 & 66 & 336 & 74 & 588 & 70 \\
\hline & $>1.8$ & 18 & 5 & 15 & 3 & 33 & 4 \\
\hline & Missing & 6 & 2 & 1 & $<1$ & 7 & 1 \\
\hline Serum creatinine & $<130$ & 268 & 71 & 392 & 86 & 660 & 79 \\
\hline \multirow[t]{3}{*}{$(\mu \mathrm{mol} / \mathrm{l})$} & 130-199 & 61 & 16 & 34 & 7 & 95 & 11 \\
\hline & $>199$ & 46 & 12 & 27 & 6 & 73 & 9 \\
\hline & Missing & 5 & 1 & 3 & 1 & 8 & 1 \\
\hline
\end{tabular}
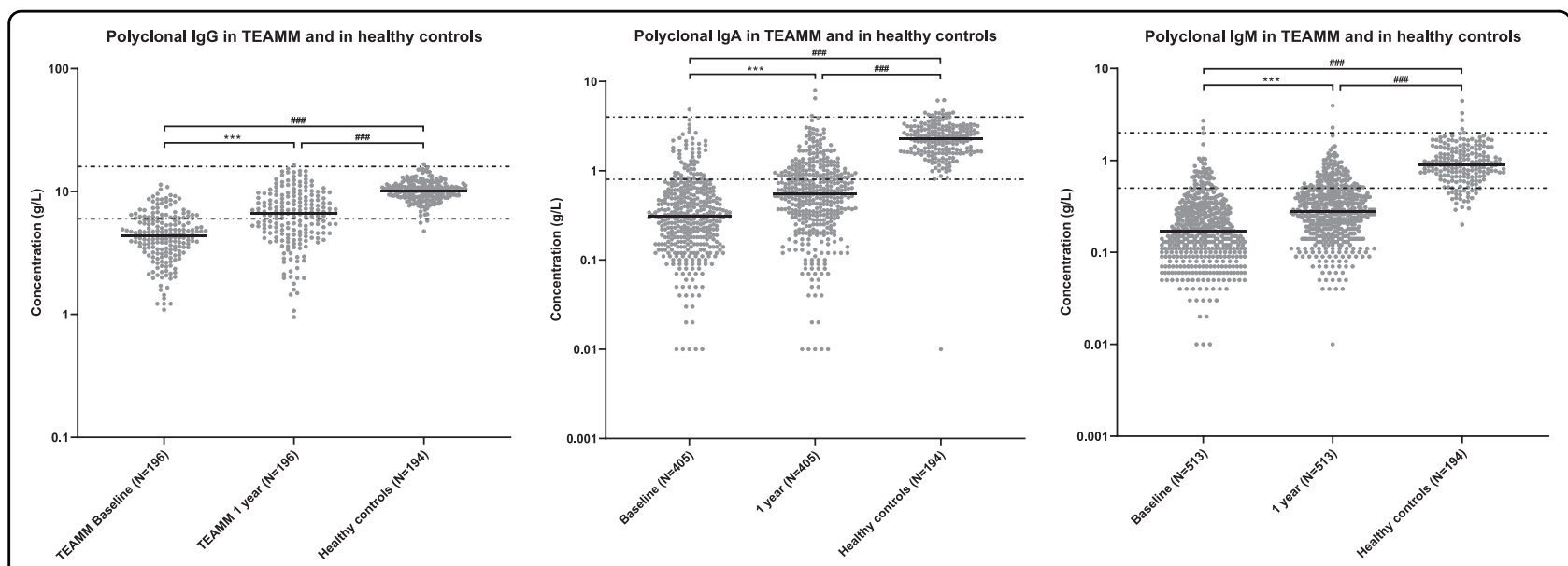

Fig. 1 Levels of polyclonal IgG, IgA and IgM in healthy controls and TEAMM patients without an IgG, IgA and IgM M-protein, respectively, at diagnosis and 1 year post-diagnosis. Distribution of polyclonal antibody quantified in a healthy cohort and TEAMM patients without an lgG, IgA and IgM M-protein, respectively, at disease presentation (baseline) and at 1 year post diagnosis. The median line for each cohort is shown in black while the 5th and 95th centile of polyclonal normal range (lgG: $6 \mathrm{~g} / \mathrm{l}$ and $16 \mathrm{~g} / \mathrm{l} ; \mathrm{lgA}: 0.8 \mathrm{~g} / \mathrm{l}$ and $4 \mathrm{~g} / \mathrm{l} ; \mathrm{lgM}: 0.5 \mathrm{~g} / \mathrm{l}$ and $2 \mathrm{~g} / \mathrm{l}$ ) are represented as discontinuous lines. Statistical significance was calculated using the Wilcoxon and the Mann-Whitney tests and is shown for each pair of data ${ }^{* * *} p<0.001$, \#\#\# $p<0.001$ ).

M-protein, respectively, at disease diagnosis and at 1 year. Polyclonal IgG, IgA and IgM levels were below the reference range (RR) in $71 \%, 83$ and $90 \%$ of $838 \mathrm{MM}$ patients at diagnosis (Fig. 1). In 196 patients with paired polyclonal IgG levels at baseline and 1 year the proportion of patients with levels within the RR increased from 25 to $57 \%$, with median levels increasing from 4.4 to $6.6 \mathrm{~g} / \mathrm{l}$. For 405 patients with paired polyclonal IgA levels the increase by 1 year was from 15 to $33 \%$ with median levels increasing from 0.3 to $0.6 \mathrm{~g} / \mathrm{l}$. For 513 patients with paired polyclonal IgM levels at both time-points the increase in proportion with levels within the RR was from $10 \%$ at diagnosis to $21 \%$ at 1 year with median levels increasing from 0.2 to $0.3 \mathrm{~g} / \mathrm{l}$ (Fig. 1). These increases in polyclonal immunoglobulin levels between diagnosis and 1 year occurred in both patients who were transplant eligible (TE) and transplant non-eligible (TNE) with no significant difference between the two cohorts $(p=.55$ for IgG, 
$p=0.57$ for IgM and $p=0.78$ for IgA (Fig. S1)). Similarly, when we split patients according to whether they were randomised to levofloxacin or placebo there was no difference in the increases in polyclonal immunoglobulin levels between diagnosis and 1 year (data not shown). For all three types of polyclonal immunoglobulins median levels in TEAMM patients at all time-points were significantly lower than median levels identified in the healthy cohort (Fig. 1).

\section{Severely reduced polyclonal immunoglobulins are associated with higher infection rates}

Infection rates and febrile episodes were analysed according to degree polyclonal immunodeficiency (normal, slightly reduced and severely reduced) and are reported in Table S1. There was no difference in incidence of infections or febrile episodes based on degree of polyclonal IgG deficiency. Infections and febrile episodes were more likely to occur $(p<0.05)$ in patients with severely reduced polyclonal IgA (47\% and $26 \%$ of patients, respectively) vs. normal polyclonal IgA (35\% and $16 \%$ of patients, respectively). Similarly, incidence of infection was significantly associated with severely reduced polyclonal IgM (48\%) vs. normal IgM (36\%), $p<0.01$; febrile episodes also occurred more frequently in patients with severely $(23 \%)$ and slightly (22\%) reduced polyclonal IgM compared to those with normal IgM levels (15\%), $p<0.05$.

\section{Anti-bacterial IgG antibody levels are very low at MM diagnosis}

At diagnosis median levels of total polyclonal $\operatorname{IgG}$ are $4.4 \mathrm{~g} / \mathrm{l}(43 \%)$ compared to $10.2 \mathrm{~g} / \mathrm{l}$ for healthy controls. Figure 2 shows the proportions of heathy volunteers and myeloma patients that have IgG antibody levels above protective thresholds for 19 bacterial antigen targets (12 pneumococcal serotypes, 4 meningococcal serotypes, haemophilus, tetanus and diphtheria toxoids). Figure 3 shows the specific antibody levels for these 838 patients. The mean percentage of the $838 \mathrm{MM}$ patients at diagnosis with protective IgG antibody levels against any one of the 19 bacterial antigen targets was $20 \%$ compared to $55 \%$ of the 193 healthy controls. Of the pneumococcal serotypes,

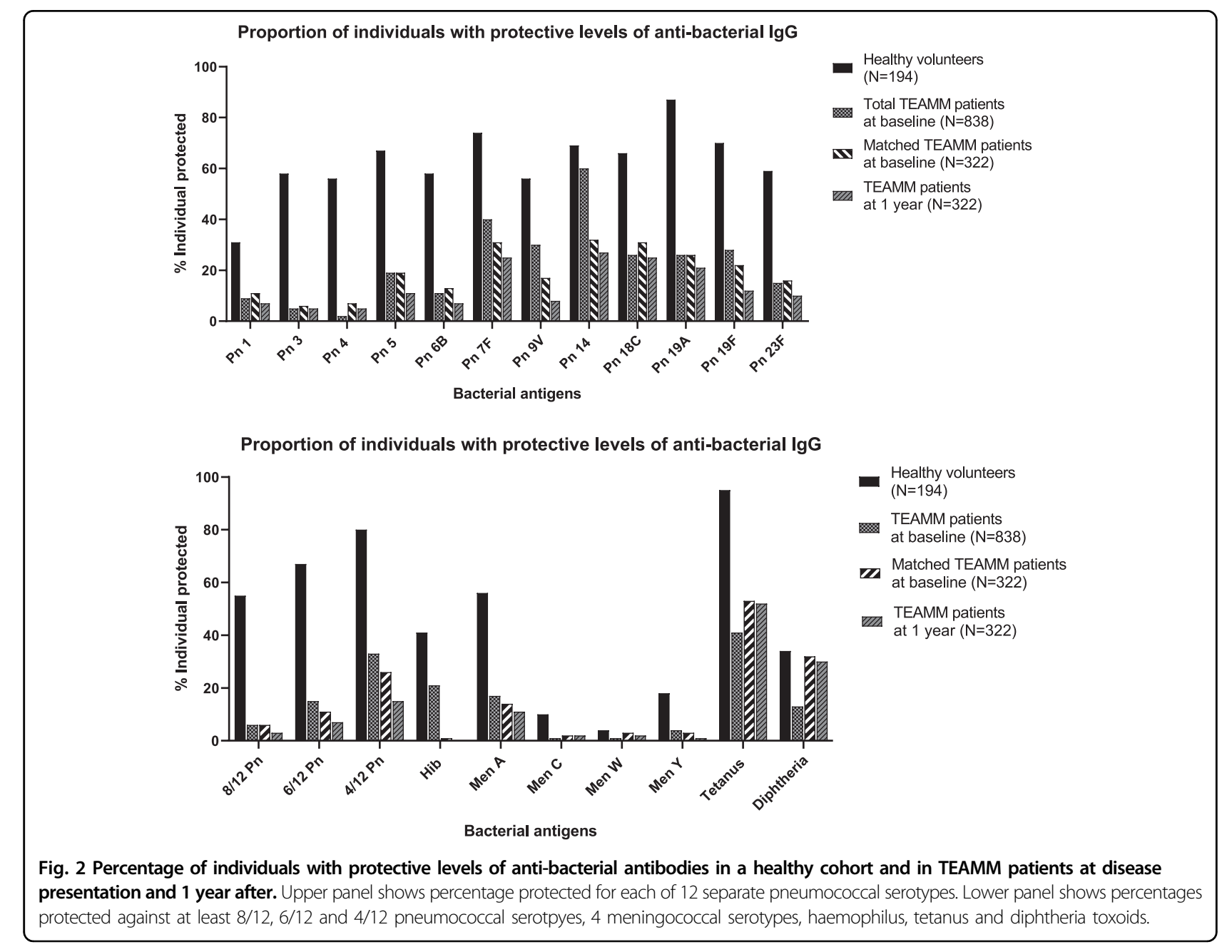




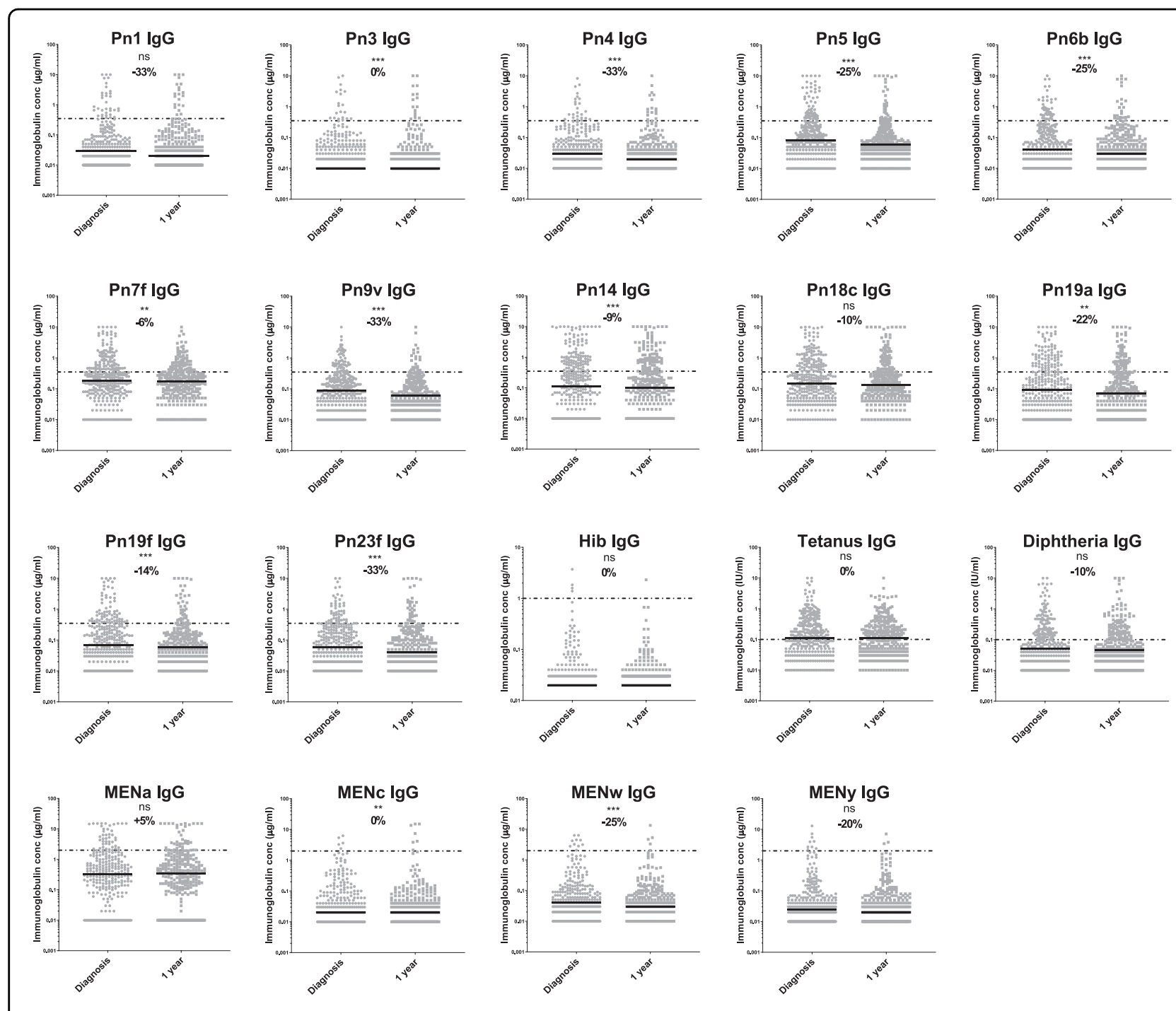

Fig. 3 Anti-bacterial serum antibody levels in TEAMM patients at diagnosis and 1 year post-diagnosis compared with protective thresholds. Distribution of anti-bacterial antibody concentrations quantified at disease presentation (baseline) and after 1 year in TEAMM patients ( $n=322$ ). The median for each antibody concentration is shown in black and the recommended lgG concentrations for protection against each bacteria is presented as discontinuous lines: $0.35 \mu \mathrm{g} / \mathrm{ml}$ for pneumococcal (Pn) polysaccharide, $2.0 \mu \mathrm{g} / \mathrm{ml}$ for meningococcal (Men) polysaccharide, $1.0 \mu \mathrm{g} / \mathrm{ml}$ for Haemophilus influenzae b (Hib), $0.1 \mathrm{IU} / \mathrm{ml}$ for tetanus and diphtheria antigens. Percentage reduction between median levels at presentation and at 1 year is shown. Statistical significance was calculated using the Wilcoxon test and is shown for each pair of data ${ }^{*} p<0.5$; ${ }^{* *} p<0.01$; ${ }^{* * *} p<0.001$; ns $p<0.5$ ).

Pn1, 3, and 4 were the most suppressed with $<10 \%$ of myeloma patients showing the minimum level required for protection. The only serotype with adequate protection was Pn14, with $60 \%$ of patients achieving the threshold of $\geq 0.35 \mu \mathrm{g} / \mathrm{ml}$ and the only bacterial antigen out of all 19 investigated with more than half of patients protected. Only $6 \%$ of myeloma patients had protective levels for $2 / 3$ rd of pneumococcal serotypes compared with $55 \%$ of healthy donors. Protective levels for half and a third of pneumococcal serotypes, respectively, were $15 \%$ and $33 \%$ in myeloma patients compared to $6 \% 7$ and $80 \%$ for healthy controls. The highest level of protection for the healthy donors was found for tetanus toxoid, where
95\% of individuals displayed protective levels in contrast to $41 \%$ of myeloma patients. A positive correlation was found between levels of polyclonal IgG $(r=0.96)$ and IgM $(r=0.84)$ at presentation and the numbers of bacterial serotypes for which specific antibody levels were protective (Fig. 4).

Antibody protection against bacterial antigens was found to differ between patients under 65 and greater or equal to 65 years old (Table 2). A higher proportion of younger myeloma patients had protective levels against Haemophilus, Meningococcal serotypes, Tetanus and Diphtheria toxoids. Conversely, a higher proportion of older patients had protective levels against pneumococcal serotypes. 

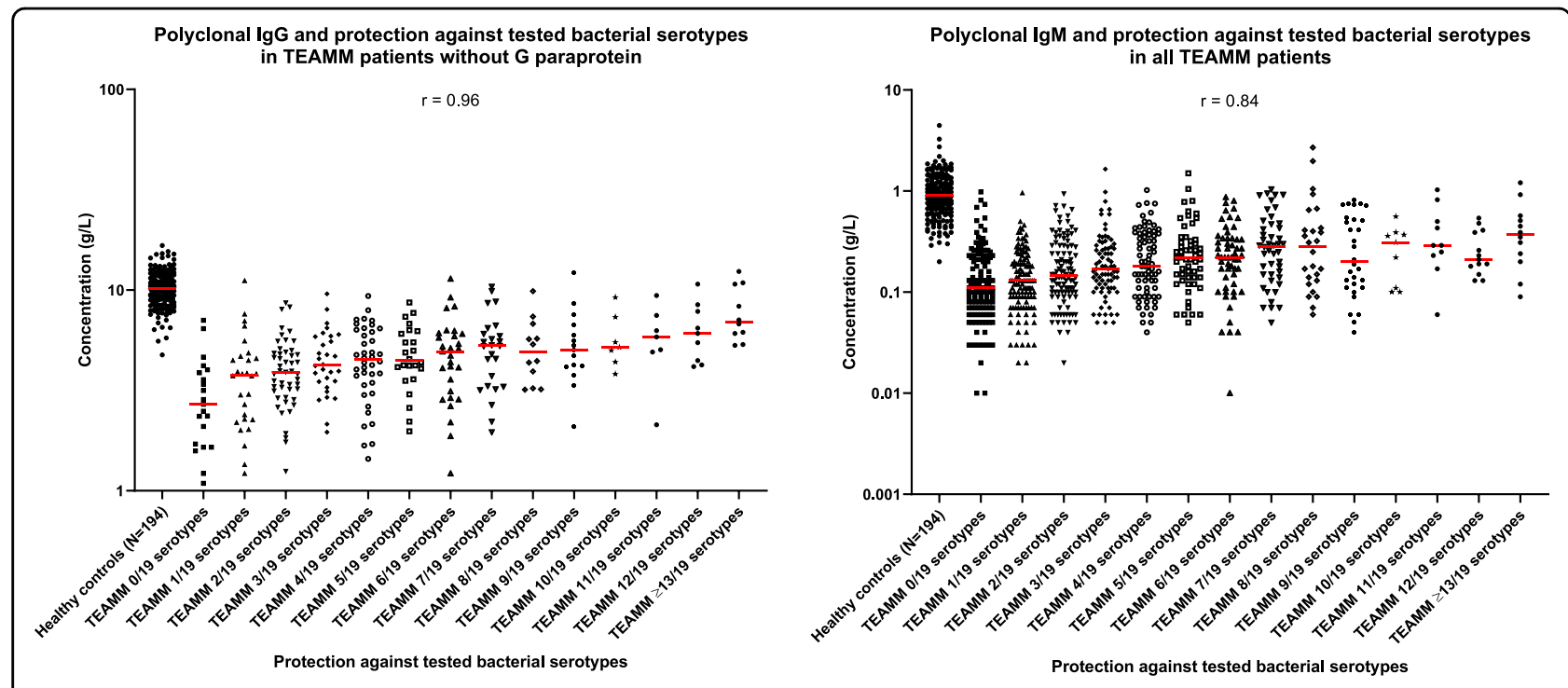

Fig. 4 Correlation between polyclonal IgG and IgM with protection against bacterial serotype in TEAMM patients at presentation and including healthy controls for reference levels. Polyclonal lgG and IgM in a healthy cohort and in TEAMM patients in correlation with their level of protection against bacterial serotypes. Median levels of polyclonal immunoglobulins are shown in red for each group. Spearman correlation coefficient is reported for each analysis.

\section{By 1 year IgG antibodies against protein antigens have changed little and against bacterial polysaccharide antigens have reduced to lower levels than seen at diagnosis}

Anti-bacterial antibodies were measured in paired samples from diagnosis and at 1 year for $322 \mathrm{MM}$ patients. The concentration of antibodies against the 19 bacterial antigens are shown in Fig. 3 and comprise the two thymus-dependent protein antigens tetanus and diphtheria toxoids and the seventeen thymus independent polysaccharide antigens, haemophilus, 4 meningococcal serotypes and 12 pneumococcal serotypes. For the two protein antigens there was no significant difference in overall concentrations of IgG anti-toxoid antibodies between baseline and 1 year (Fig. 3), although antibody median titres were significantly increased at 1 year compared to baseline for both toxins when patients were divided by treatment in TE and TNE (30\% in TNE and $63 \%$ in TE for tetanus and $100 \%$ in TNE and 33\% in TE for diphtheria (Fig. S2)). No significant differences were identified in the levels of anti-toxoid antibodies at baseline between TE and TNE ( $p=0.17$ for tetanus and $p=0.11$ for diphtheria), while anti-tetanus levels were $44 \%$ higher at 1 year in TE patients compared to TNE (Fig. S2). There was no difference in the proportions of patients protected at baseline and 1 year for tetanus (53 and 52\%) or diphtheria (32 and 30\%) toxins (Fig. 2). In contrast levels of antibody against polysaccharide antigens fell by a year both as median titres (Fig. 3) and proportion of patients protected (Fig. 2). For haemophilus and meningococcal C, $\mathrm{W}$ and $\mathrm{Y}$ serotypes most patients had antibody levels
50-100 times lower than the protective threshold and there was little difference between diagnosis and a year. For meningococcal A and the pneumococcal serotypes there was a reduction in antibodies between diagnosis and a year. There were significant reductions in IgG titres between diagnosis and a year for pneumococcal serotypes 3, 4, 5, 6b, 7f, 9v, 14, 19a, 19f, and 23f (Fig. 3). The percentage of people protected against 4/12 serotypes reduced from 26 to 15 and for meningococcal A from 14 to $11 \%$. No significant differences were found between levofloxacin treatment or placebo for antibody levels or percentage protected at 1 year.

\section{Prediction of time to febrile episode or death within 12 weeks of starting trial treatment}

No relationships were found between levels of protection against the 19 bacterial serotypes and the occurrence of deaths, febrile infections, non-febrile infections or total infections in the first 12 weeks from entry to the trial (Table S2). Cox regression analysis showed that use of prophylactic septrin was the single most important predicator of time to febrile episode or death within 12 weeks of starting trial treatment. Septrin retained significance in a multivariate model adjusting for baseline factors and antigens. A cox regression model excluding septrin identified ECOG performance status as the only factor with borderline significance (Table S3). Neither polyclonal IgM, nor the levels of specific antibody against the 17 polysaccharide and two protein bacterial targets were significant predicators of febrile episode or death (Table S3). 
Table 2 Percentage of individuals with protective levels of anti-bacterial antibodies for TEAMM patients $<$ or $\geq 65$ years old.

\begin{tabular}{|c|c|c|}
\hline \multirow[b]{2}{*}{$\begin{array}{l}\text { Antigens (protective } \\
\text { threshold) }\end{array}$} & \multicolumn{2}{|c|}{$\begin{array}{l}\text { Proportion of individuals with protective } \\
\text { levels of anti-bacterial lgGs }\end{array}$} \\
\hline & $\begin{array}{l}\text { MM patients Under } \\
65(n=335)\end{array}$ & $\begin{array}{l}\text { MM patients } 65 \text { and } \\
\text { over }(n=503)\end{array}$ \\
\hline Pn $1(\geq 0.35 \mu \mathrm{g} / \mathrm{ml})$ & 4.6 & $12.4^{* * * *}$ \\
\hline Pn $3(\geq 0.35 \mu \mathrm{g} / \mathrm{ml})$ & 4.6 & 4.1 \\
\hline Pn $4(\geq 0.35 \mu \mathrm{g} / \mathrm{ml})$ & 0.9 & 2.4 \\
\hline Pn $5(\geq 0.35 \mu \mathrm{g} / \mathrm{ml})$ & 14.0 & $23.0^{* *}$ \\
\hline $\operatorname{Pn} 6 \mathrm{~b}(\geq 0.35 \mu \mathrm{g} / \mathrm{ml})$ & 9.7 & 11.5 \\
\hline $\operatorname{Pn} 7 \mathrm{~F}(\geq 0.35 \mu \mathrm{g} / \mathrm{ml})$ & 38.3 & 40.6 \\
\hline Pn 9 V $(\geq 0.35 \mu \mathrm{g} / \mathrm{ml})$ & 27.4 & 31.4 \\
\hline Pn $14(\geq 0.35 \mu \mathrm{g} / \mathrm{ml})$ & 58.9 & 62.3 \\
\hline Pn $18 \mathrm{C}(\geq 0.35 \mu \mathrm{g} / \mathrm{ml})$ & 17.1 & $31.5^{* * * *}$ \\
\hline Pn 19 A $(\geq 0.35 \mu \mathrm{g} / \mathrm{ml})$ & 22.6 & 27.8 \\
\hline Pn $19 \mathrm{~F}(\geq 0.35 \mu \mathrm{g} / \mathrm{ml})$ & 24.6 & 29.3 \\
\hline $\operatorname{Pn} 23 \mathrm{~F}(\geq 0.35 \mu \mathrm{g} / \mathrm{ml})$ & 13.1 & 16.0 \\
\hline 8/12 Pn Serotypes & 4.6 & 6.5 \\
\hline 6/12 Pn Serotypes & 10.9 & $18.4^{* *}$ \\
\hline 4/12 Pn Serotypes & 28.6 & 34.8 \\
\hline $\mathrm{Hib}(\geq 1 \mu \mathrm{g} / \mathrm{ml})$ & 22.0 & 21.0 \\
\hline Tetanus ( $\geq 0.1 \mathrm{IU} / \mathrm{ml}$ ) & 46.0 & $36.7^{* *}$ \\
\hline Diphtheria ( $\geq 0.1 \mathrm{IU} / \mathrm{ml}$ ) & 13.7 & 13.0 \\
\hline Men A $(\geq 2 \mu \mathrm{g} / \mathrm{ml})$ & 17.7 & 16.9 \\
\hline Men C ( $\geq 2 \mu \mathrm{g} / \mathrm{ml})$ & 1.4 & $0.2^{*}$ \\
\hline Men W $(\geq 2 \mu \mathrm{g} / \mathrm{ml})$ & 1.7 & 1.1 \\
\hline Men $Y(\geq 2 \mu \mathrm{g} / \mathrm{ml})$ & 4.9 & 4.3 \\
\hline
\end{tabular}

The italicised values are used to indicate higher percentages of individual with protective levels between the two age groups. Statistical significance was calculated using the Chi-square test and is shown for $<65$ vs. $\geq 65$ years old myeloma patients $\left({ }^{*} p<0.05 ;{ }^{* *} p<0.01 ;{ }^{* * *} p<0.001\right)$.

\section{Discussion}

Polyclonal serum IgG, IgA and IgM levels were low at myeloma diagnosis as we and others have found previously $^{11-13}$. This immunoparesis is present in $85 \%$ of patients and is severe. In our previous study we found immunoparesis, particularly of IgM, associated with adverse PFS and OS but not strongly associated with infection and this has been reported by other groups $^{12,13,25}$. In the TEAMM study, infection was a major outcome in the first 12 weeks from diagnosis when infection is commonest and so this provided a good platform for more detailed investigation of the utility of polyclonal immunoglobulin levels for predicting risk of infection. Further, for the first time, we have been able to investigate, in a large number of MM patients, specific titres of antibody against a range of bacterial antigens (functional antibody) to predict infection and guide use of management strategies including prophylactic antibiotics. We show functional antibodies are even more severely compromised at disease presentation than total polyclonal antibody levels although the two are correlated.

Levels of functional antibody against the thymusdependent antigen tetanus toxoid were the least impaired with $41 \%$ of patients protected vs. $95 \%$ of healthy controls. Following pneumococcal vaccination achievement of protective levels of antibody against two thirds of serotypes tested is considered a satisfactory antibody response. Most of the healthy donors would not have received a pneumococcal vaccination but by natural exposure to pneumococci $55 \%$ had protective antibody levels against $8 / 12$ serotypes vs. just $6 \%$ of the myeloma patients.

The proportion of myeloma patients with protective antibody levels against tetanus toxoid was significantly lower in older myeloma patients as expected because of immunosenesence. Conversely, a higher proportion of older patients had protective levels against pneumococcal serotypes. This may reflect the UK 23-valent polysaccharide pneumococcal vaccination (PPV23) programme for individuals aged above $65^{26}$. Although these findings provide some support for the efficacy of the current vaccination programme in this age/patient population, significant differences were only found for 4 pneumococcal serotypes. Further, the majority of older patients still failed to meet protective levels and only $6.5 \%$ of older myeloma patients demonstrated protective levels for $8 / 12$ or more serotypes. Previous studies have shown reduced levels of antibody production to PPV23 in MM patients ${ }^{7,27}$.

Higher proportions of patients were found to experience infections and febrile episodes when polyclonal IgA and IgM were severely reduced but not IgG. However, cox regression analysis showed total polyclonal antibody levels nor titres of antibody against 19 different bacterial antigenic targets predicted infections and deaths in the first twelve weeks from diagnosis. This may reflect that stratification by immunoparesis at diagnosis is limited because the great majority of MM patients have severe immunoparesis whether assessed by total or specific functional antibody levels. Additionally, they have a broad and profound immunodeficiency encompassing reduced integrity of barriers to infection and major deficits in other components of adaptive immunity and of innate immunity. The greater importance of this much broader immunodeficiency causal to high infection rates is supported by lack of efficacy of IgG replacement therapy in newly diagnosed myeloma. In a double-blind placebo-controlled 
trial in 203 newly diagnosed myeloma patients infusions of placebo or $18 \mathrm{~g}$ human polyclonal IgG on days $1,8,22$, 43, 64 and 85 from entry to the study did not alter the incidence or type of infections and did not alter hospital admissions or antibiotic use for infections (MacLennan ICM personal communication. A Trial of Intravenous Immunoglobulin Prophylaxis in Myelomatosis for the MRC Working Party on Leukaemia in adults. Presented to the 1993 UK annual review).

There is a gradation of severity of immunoparesis from Monoclonal Gammopathy of Undetermined Significance (MGUS) to Smouldering MM and through increasing severity of MM stages ${ }^{12,17}$. Accordingly as anti-myeloma therapy brings patients into remission one might expect a degree of recovery from immunoparesis particularly as current myeloma treatments induce complete responses in higher proportions of patients. Post high-dose melphalan and autologous stem cell transplant about half of myeloma patients have recovery of normal levels of polyclonal immunoglobulins and these patients compared to those with sustained immunoparesis have improved PFS and $\mathrm{OS}^{28-30}$. We have found the same in our TE patients and importantly also (we believe for first time) that TNE patients had a similar recovery of polyclonal immunoglobulin levels post induction treatment. This observation is limited in being made at the fixed time point of 1 year from entry to the trial and not all patients will have completed their TE or TNE treatments and reached remission. A further limitation is that the trial did not extend observations beyond a year and so we have not been able to assess recovery from immunoparesis as a prognostic factor for PFS or OS.

Despite substantial recovery of total polyclonal IgG levels at 1 year in both TE and TNE patients, median titres and percentage of patients with protective IgG levels did not increase for tetanus and diphtheria toxins. Further, levels of specific IgG against bacterial polysaccharides (12 pneumococcal serotypes, 4 meningococcal serotypes and haemophilus) had fallen from very low levels at diagnosis to levels, suggesting their immune systems had reverted to a naive state. For most patients and against most bacterial antigen targets levels of antibody were 50 - to a 100-fold lower than protective levels.

The findings of this study show that functional antibody levels against certain pathogens worsens following treatment and does not mirror total immunoglobulin recovery. Presumably this reflects recovery of humoral immunity following myeloma therapy being skewed by the antigenic and cytokine environment at that time and would not favour recovery of humoral immunity to pathogens to which the individual was vaccinated against or exposed against much earlier in life.

In conclusion, these data demonstrate the need to protect patients against infections at diagnosis, during anti-myeloma therapy and importantly also during remission phases. Prophylactic antibiotics and patient education, should be considered during active disease/ when patients are undergoing therapy. The TEAMM trial showed a clear benefit for levofloxacin in the first 12 weeks and further studies are needed to identify optimal prophylaxis (levofloxacin, septrin, or a combination of both) and the optimal duration for prophylaxis. Vaccination should be avoided while patients are on treatment but this study clearly demonstrates the need for vaccination following treatment: in remission despite substantial recovery of total polyclonal immunoglobulin levels patient's humoral immune system has been rendered naive by the disease and its treatment meaning they lack protective antibody levels against most bacterial antigens.

Current data on vaccination in myeloma is limited and this study highlights the necessity to determine optimal vaccination timing and need for booster vaccinations following treatment. The European Myeloma Network currently recommendations using a broad spectrum of vaccinations to overcome severely reduced antibody titres associated with autologous and particularly allogenic transplantation ${ }^{31}$. However, these guidelines largely reflect findings in the general/elderly population or are based on risk of disease. Consequently there is a need to design and conduct trials to investigate optimal timing and vaccination strategies in myeloma patients, specifically in relation to bacteria (such as pneumococcal, mennigocci) and also viruses (influenza) that pose a high risk to myeloma patients. The efficacy of vaccination schedules in remission should be considered and tested for all myeloma patients both in individual patients through measurement of antibody response to vaccination and in studies comparing different vaccination schedules.

\section{Acknowledgements \\ The TEAMM trial and clinical data collection was coordinated by Warwick Clinical Trials Unit and the translational work carried out at the Clinical Immunology Service at the University of Birmingham. This research was funded by the Efficacy and Mechanism Evaluation (EME) Programme, an MRC and NIHR partnership. The TEAMM trial was funded by the National Institute for Health Research (NIHR) Health Technology Assessment Programme (grant no 08/116/69). The views expressed in this publication are those of the author(s) and not necessarily those of the MRC, NHS, NIHR or the UK Department of Health. We thank the patients who were recruited into this study and their families and the clinicians and staff at each centre who looked after these patients.}

\footnotetext{
Author details

${ }^{1}$ Institute of Immunology and Immunotherapy, Clinical Immunology Service, University of Birmingham, Birmingham, UK. ${ }^{2}$ Warwick Clinical Trial Unit, University of Warwick, Coventry, UK. ${ }^{3}$ King's College Hospital NHS Trust, London, UK. ${ }^{4}$ University Hospital Birmingham NHS Trust, Birmingham, UK. ${ }^{5}$ Department of Haematology, UCL Cancer Institute, London, UK. 'St. George's Hospital, University of London, Department of Medical Microbiology, Tooting, London, UK
} 


\section{Conflict of interest}

M.T.D. reports personal fees from Abingdon Health Ltd (York, UK), outside the submitted work. All other authors declare no competing interests.

\section{Publisher's note}

Springer Nature remains neutral with regard to jurisdictional claims in published maps and institutional affiliations.

Supplementary Information accompanies this paper at (https://doi.org/ 10.1038/s41408-020-00370-7).

Received: 9 June 2020 Revised: 15 September 2020 Accepted: 28 September 2020

Published online: 04 November 2020

\section{References}

1. Kyle \& Rajkumar, S. V. Multiple myeloma. N. Engl. J. Med. 351, 1860-1873 (2004).

2. Palumbo, A., Anderson, K. \& Battista, S. G. Multiple Myeloma. N. Engl. J. Med. [Internet] 364, 1046-1060 (2011).

3. Cancer Research UK. Myeloma incidence statistics|Cancer Research UK [Internet]. https:/www.cancerresearchuk.org/health-professional/cancer-statistics/ statistics-by-cancer-type/myeloma.

4. Kumar, S. K. et al. Continued improvement in survival in multiple myeloma: changes in early mortality and outcomes in older patients. Leukemia $\mathbf{2 8}$, 1122-1128 (2014)

5. Blimark, C. et al. Multiple myeloma and infections: A population-based study on 9253 multiple myeloma patients. Haematologica 100, 107-113 (2015).

6. Noone, A. et al. SEER Cancer Statistics Review, 1975-2015. https://seer.cancer.gov/ csr/1975_2015/, based on November 2017 SEER data submission, posted to the SEER web site, April 2018. [Internet]. SEER Cancer Statistics Review, 1975-2013 (National Cancer Institute, Bethesda, MD, 2015).

7. Nucci, M. \& Anaissie, E. Infections in patients with multiple myeloma in the era of high-dose therapy and novel agents. Clin. Infect. Dis. 49, 1211-1225 (2009).

8. McShane, C. M., Murray, L. J., Engels, E. A., Landgren, O. \& Anderson, L. A. Common community-acquired infections and subsequent risk of multiple myeloma: A population-based study. Int. J. Cancer 134, 1734-1740 (2014).

9. Augustson, B. M. et al. Early mortality after diagnosis of multiple myeloma: Analysis of patients entered onto the United Kingdom Medical Research Council trials between 1980 and 2002-Medical Research Council Adult Leukaemia Working Party. J. Clin. Oncol. 23, 9219-9226 (2005).

10. Holmström, M. O. et al. Causes of early death in multiple myeloma patients who are ineligible for high-dose therapy with hematopoietic stem cell support: A study based on the nationwide Danish Myeloma Database. Am. J. Hematol. 90, E73-E74 (2015).

11. Wangel, A. Multiple myeloma and polyclonal hypogammaglobulinaemia. Acta Med. Scand. 221, 421-425 (1987)

12. Heaney, J. L. J. et al. Characterisation of immunoparesis in newly diagnosed myeloma and its impact on progression-free and overall survival in both old and recent myeloma trials. Leukemia 32, 1727-1738 (2018).

13. Kastritis, E. et al. Preserved levels of uninvolved immunoglobulins are independently associated with favorable outcome in patients with symptomatic multiple myeloma. Leukemia 28, 2075-2079 (2014).
14. Hargreaves, R. M. et al. Immunological factors and risk of infection in plateau phase myeloma. J. Clin. Pathol. 48, 260-266 (1995).

15. Sørrig, R., Klausen, T. W., Salomo, M., Vangsted, A. \& Gimsing, P. Risk factors for blood stream infections in multiple myeloma: A population-based study of 1154 patients in Denmark. Eur. J. Haematol. 101, 21-27 (2018).

16. Sørrig, R., Klausen, T. W., Salomo, M., Vangsted, A. \& Gimsing, P. Risk factors for infections in newly diagnosed Multiple Myeloma patients: a Danish retrospective nationwide cohort study. Eur. J. Haematol. 102, 182-190 (2019).

17. Pruzanski, W., Gidon, M. S. \& Roy, A. Suppression of polyclonal immunoglobulins in multiple myeloma: Relationship to the staging and other manifestations at diagnosis. Clin. Immunol. Immunopathol. 17, 280-286 (1980).

18. Drayson, M. T. et al. Levofloxacin prophylaxis in newly diagnosed myeloma patients. Lancet (London, England). 2019.

19. Whitelegg, A. M. E. et al. Measurement of antibodies to pneumococcal, meningococcal and haemophilus polysaccharides, and tetanus and diphtheria toxoids using a 19-plexed assay. J. Immunol. Methods 377, 37-46 (2012).

20. WHO/Health Canada consultation on serological criteria for evaluation and licensing of new pneumococcal vaccines. World Health Organisation. https:// www.who.int/biologicals/publications/meetings/areas/vaccines/ pneumococcal/en/ (2008).

21. Peltola, $H$. et al. Clinical efficacy of meningococcus group A capsular polysaccharide vaccine in children three months to five years of age. $N$. Engl. J. Med. 297, 686-691 (1977)

22. Käyhty, H., Peltola, H., Karanko, V. \& Mäkelä, P. H. The protective level of serum antibodies to the capsular polysaccharide of Haemophilus influenzae type b. J. Infect. Dis. 147, 1100 (1983).

23. Plotkin, S. A. Immunologic correlates of protection induced by vaccination. Pediatr. Infect. Dis. J. 20, 63-75 (2001)

24. Drayson, M. T. et al. Prophylactic levofloxacin to prevent infections in newly diagnosed symptomatic myeloma: The TEAMM RCT. Health Technol. Assess. (Rockv) 23, 1-94 (2019).

25. Sørrig, R. et al. Immunoparesis in newly diagnosed Multiple Myeloma patients: effects on overall survival and progression free survival in the Danish population. PLOS ONE 12,1-15 (2017).

26. Immunisation Department P. Pneumococcal Polysacchride Vaccine (PPV) coverage report, England, April 2018 to March 2019. https://assets.publishing.service. gov.uk/government/uploads/system/uploads/attachment_data/file/847408/ hpr3919 PPV.pdf. (Public Health England, 2019).

27. Karlsson, J. et al. Comparative study of immune status to infectious agents in elderly patients with multiple myeloma, Waldenstrom's macroglobulinemia, and monoclonal gammopathy of undetermined significance. Clin. Vaccine Immunol. 18, 969-977 (2011)

28. González-Calle, V. et al. Recovery of polyclonal immunoglobulins one year after autologous stem cell transplantation as a long-term predictor marker of progression and survival in multiple myeloma. Haematologica 102, 922-931 (2017).

29. Jimenez-Zepeda, V. H. et al. Immunoparesis and polyclonal immunoglobulin recovery after auto-SCT for patients with multiple myeloma treated at a single institution. Leuk. Lymphoma 59, 1920-1926 (2018).

30. Gao, W. et al. Immunoparesis recovery 1 year after ASCT is independently associated with favorable survival in patients with symptomatic multiple myeloma who undergo autologous stem cell transplantation. Ann. Hematol. 98, 1177-1184 (2019)

31. Ludwig, $\mathrm{H}$. et al. Recommendations for vaccination in multiple myeloma: a consensus of the European Myeloma Network. Leukemia 2020 https://doi.org/ $10.1038 / s 41375-020-01016-0$ 